Open Access

\title{
Complete genome sequencing of Dehalococcoides sp. strain UCH007 using a differential reads picking method
}

\author{
Yoshihito Uchino', Takamasa Miura ${ }^{1 *}$, Akira Hosoyama', Shoko Ohji ${ }^{1}$, Atsushi Yamazoe ${ }^{1}$, Masako Ito²,
} Yoh Takahata ${ }^{2}$, Ken-ichiro Suzuki ${ }^{1}$ and Nobuyuki Fujita ${ }^{1}$

\begin{abstract}
A novel Dehalococcoides sp. strain UCH007 was isolated from the groundwater polluted with chlorinated ethenes in Japan. This strain is capable of dechlorinating trichloroethene, cis-1,2-dichloroethene and vinyl chloride to ethene. Dehalococcoides bacteria are hardly cultivable, so genome sequencing has presented a challenge. In this study, we developed a differential reads picking method for mixed genomic DNA obtained from a co-culture, and applied it to the sequencing of strain UCH007. The genome of strain UCH0O7 consists of a 1,473,548-bp chromosome that encodes 1509 coding sequences including 29 putative reductive dehalogenase genes. Strain UCH007 is the first strain in the Victoria subgroup found to possess the pceA, tceA and vcrA genes.
\end{abstract}

Keywords: Dehalococcoides, Differential reads picking method, Bioremediation, Reductive dechlorination, Dehalorespiring, Chloroethene

\section{Introduction}

Chloroethenes such as PCE, TCE cis-1,2-DCE and VC in contaminated soil and groundwater can be removed by reductive dechlorination mediated by anaerobic bacteria. Under anaerobic conditions, dehalorespiring bacteria dechlorinate chloroethenes by mediating the step-wise replacement of chlorine with hydrogen resulting in the conversion of PCE to TCE, DCE isomers, VC, and ethene sequentially. Among many dehalorespiring bacterial isolates, only a few strains of the genus Dehalococcoides completely convert chloroethenes to nontoxic ethene, hence they are indispensable for successful bioremediation applications [1-10]. The RDases are essential enzymes for the dehalorespiring activities of Dehalococcoides ssp., however, the constitution of RDase genes in each strain varies significantly, resulting in varied dechlorination activities among strains. Among the RDase genes, $v c r A$ and $b v c A$, which dechlorinate $\mathrm{VC}$ to ethene are essential for complete dechlorination.

\footnotetext{
*Correspondence: miura-takamasa@nite.go.jp

'Biological Resource Center, National Institute of Technology and Evaluation, 2-10-49 Nishihara, Tokyo 151-0066, Japan

Full list of author information is available at the end of the article
}

In our previous report, we constructed a chloroethenedechlorinating microbial consortium derived from chloroethene-polluted groundwater in Japan, and identified some operational taxonomic units that were assigned to Dehalococcoides by amplicon sequencing of $16 \mathrm{~S}$ rRNA genes [11]. In this report, we describe a Dehalococcoides bacterium designated strain $\mathrm{UCH} 007$ isolated from the consortium, and present its complete genome sequence. Strain UCH007, the first Dehalococcoides strain isolated in Japan, was phylogenetically affiliated with the Victoria subgroup of the Dehalococcoides.

\section{Organism information}

\section{Classification and features}

A cis-1,2-DCE-to-ethene dechlorinating enrichment culture was obtained from the microbial consortium [11] by sequentially transferring to fresh media amended with acetate plus $\mathrm{H}_{2}-\mathrm{CO}_{2}(80 \%: 20 \%$, vol/vol) in the headspace and cis-1,2-DCE as the electron acceptor. Following repeated transfers to cis-1,2-DCE amended media in the presence of ampicillin or 2-bromoethanesulfonate, several series of dilution-to-extinction culturing and several agar shake processes were performed, and strain $\mathrm{UCH007}$ was obtained in pure culture. 


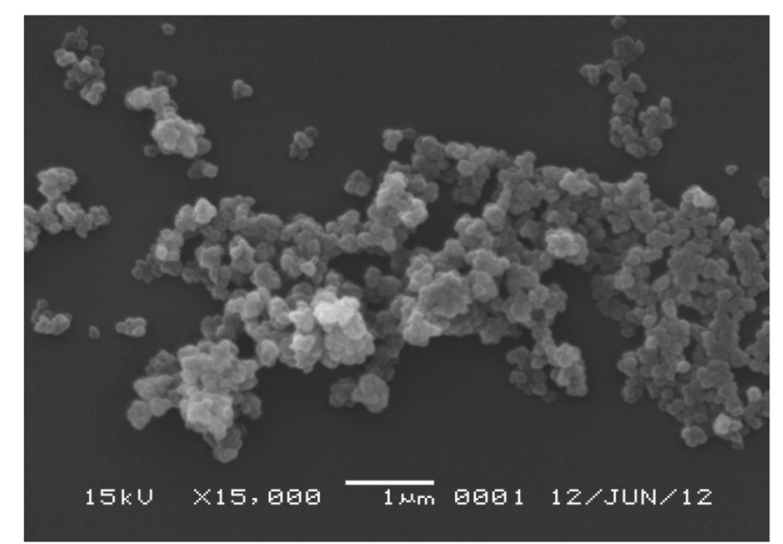

Fig. 1 Scanning electron microscopy (SEM) of Dehalococcoides sp. strain UCH007. The image was recorded using a JEOL JSM-6060 SEM (JEOL, Tokyo, Japan)

The cells of strain UCH007 were non-motile, nonspore forming and had a disc-shaped morphology with a diameter of $0.1-0.3 \mu \mathrm{m}$ (Fig. 1). The temperature range for growth of strain UCH007 was between 15 and $35{ }^{\circ} \mathrm{C}$, with optimum growth between 25 and $30{ }^{\circ} \mathrm{C}$. The $\mathrm{pH}$ range for growth of strain $\mathrm{UCH} 007$ was between 6.2 and
7.7, with an optimum $\mathrm{pH}$ between 7.0 and 7.3. The range of $\mathrm{NaCl}$ concentrations that allowed for growth of strain UCH007 was $0-1.5 \%$, with an optimum concentration of $0.3-0.5 \%$.

Strain UCH007 is a strictly anaerobic bacterium, and its growth depends on the presence of hydrogen as an electron donor, reductive dechlorination substrates such as TCE, cis-1,2-DCE, 1,1-DCE and VC as electron acceptors and acetate as a carbon source. Vitamin $B_{12}$ is essential for growth. The strain was observed to accumulate varying amounts of VC during TCE (or cis-1,2-DCE)-to-ethene dechlorination, but growth tended to be coupled with the reductive dechlorination of $\mathrm{VC}$.

Dehalococcoides strains isolated to date shared more than $98 \% 16 \mathrm{~S}$ rRNA gene sequence similarity with each other, and grouped into three subgroups designated the Pinellas, Victoria and Cornell subgroups [1]. Phylogenetic analysis based on 16S rRNA gene sequences shows that strain UCH007 belonged to the Victoria subgroup, and the most closely related strain was $D$. mccartyi strain VS with $99.92 \%$ similarity (Fig. 2). The most distantly related strain was $D$. mccartyi strain CBDB1 with $98.91 \%$ similarity.

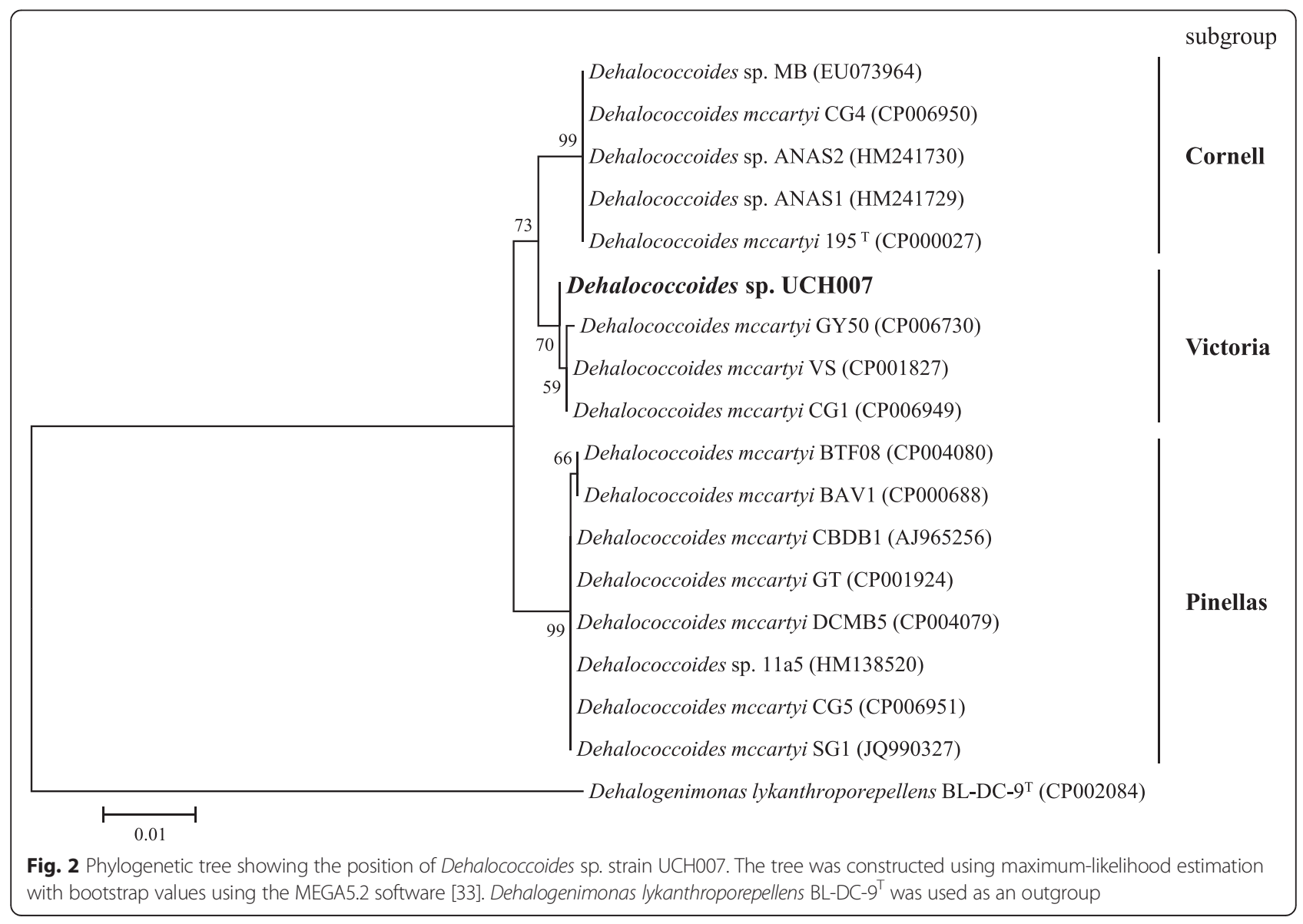




\section{Genome sequencing information Genome project history}

Strain UCH007 is the first Dehalococcoides isolate from Japan and is one of the few strains found to convert toxic chloroethenes to nontoxic ethene. It was selected for sequencing on the basis of its rarity and importance in bioremediation. Table 1 presents the project information and its association with MIGS version 2.0 compliance [12]. A summary of the project information is shown in Table 2.

\section{Growth conditions and genomic DNA preparation}

Strain UCH007 was pure-cultured in $300 \mathrm{~mL}$ of bicarbonate-buffered medium supplemented with $10 \mu \mathrm{M}$ of

Table 1 Classification and general features of Dehalococcoides sp. strain UCH0O7 [12]

\begin{tabular}{|c|c|c|c|}
\hline MIGS ID & Property & Term & $\begin{array}{l}\text { Evidence } \\
\text { code }\end{array}$ \\
\hline & \multirow[t]{8}{*}{ Classification } & Domain Bacteria & TAS [34] \\
\hline & & Phylum "Chloroflexi" & $\operatorname{TAS}[35,36]$ \\
\hline & & Class Dehalococcoidia & TAS [1] \\
\hline & & Order Dehalococcoidales & TAS [1] \\
\hline & & Family Dehalococcoidaceae & TAS [1] \\
\hline & & Genus Dehalococcoides & TAS [1] \\
\hline & & Species Dehalococcoides sp. & TAS [1] \\
\hline & & $\begin{array}{l}\text { Strain UCH007 (Taxonomy } \\
\text { ID: 1522671) }\end{array}$ & \\
\hline & Gram stain & Gram-indifferent & TAS [1] \\
\hline & Cell shape & Disk shape & IDA \\
\hline & Motility & Non-motile & IDA \\
\hline & Sporulation & Non sporulation & IDA \\
\hline & Temperature range & $15-35^{\circ} \mathrm{C}$ & IDA \\
\hline & $\begin{array}{l}\text { Optimum } \\
\text { temperature }\end{array}$ & $25-30^{\circ} \mathrm{C}$ & IDA \\
\hline & pH range; Optimum & $6.2-7.7 ; 7.0-7.3$ & IDA \\
\hline & Carbon source & Acetate & IDA \\
\hline MIGS-6 & Habitat & Groundwater & IDA \\
\hline MIGS-6.3 & Salinity & $0-1.5 \% \mathrm{NaCl}(\mathrm{w} / \mathrm{v})$ & IDA \\
\hline MIGS-22 & Oxygen requirement & Anaerobic & IDA \\
\hline MIGS-15 & Biotic relationship & Free living & TAS [1] \\
\hline MIGS-14 & Pathogenicity & None & NAS \\
\hline MIGS-4 & Geographic location & Japan & IDA \\
\hline MIGS-5 & Sample collection & 2009 & IDA \\
\hline MIGS-4.1 & Latitude & undisclosed & IDA \\
\hline MIGS-4.2 & Longitude & undisclosed & IDA \\
\hline MIGS-4.4 & Altitude & -2.5 to $-11.0 \mathrm{~m}$ & IDA \\
\hline
\end{tabular}

Evidence codes - IDA inferred from direct assay, TAS traceable author statement (i.e., a direct report exists in the literature), NAS non-traceable author statement (i.e., not directly observed for the living, isolated sample, but based on a generally accepted property for the species, or anecdotal evidence). These evidence codes are from the Gene Ontology project [37]
Table 2 Project information

\begin{tabular}{|c|c|c|}
\hline MIGS ID & Property & Term \\
\hline MIGS 31 & Finishing quality & Finished \\
\hline MIGS-28 & Libraries used & $\begin{array}{l}454 \text { standard library and Illumina } \\
\text { MiSeq library (paired-end) }\end{array}$ \\
\hline MIGS 29 & Sequencing platforms & $\begin{array}{l}454 \text { GS FLX Titanium, Illumina } \\
\text { MiSeq }\end{array}$ \\
\hline \multirow[t]{2}{*}{ MIGS 31.2} & Fold coverage & $20.25 \times 454$ GS FLX Titanium \\
\hline & & $98.35 \times$ Illumina MiSeq \\
\hline MIGS 30 & Assemblers & Newbler 2.6 \\
\hline \multirow[t]{6}{*}{ MIGS 32} & Gene calling method & MiGAP \\
\hline & Genome database release & DDBJ \\
\hline & Locus Tag & UCH0O7 \\
\hline & Genbank ID & AP014722 \\
\hline & Genbank Date of Release & February 15, 2015 \\
\hline & BIOPROJECT & PRJDB2892 \\
\hline \multirow[t]{2}{*}{ MIGS 13} & Source Material Identifier & UCH007 \\
\hline & Project relevance & $\begin{array}{l}\text { The microbial biodegradation of } \\
\text { pollutants is attracting attention } \\
\text { to find feasible ways to clean-up } \\
\text { contaminated environments. }\end{array}$ \\
\hline
\end{tabular}

cis-1,2-DCE for 47 days [3], however, the number of cells was insufficient for genome sequencing using nextgeneration sequencers. So, WGA using the pure culture as a template was performed using the REPLI-g Mini Kit (Qiagen GmbH, Hilden, Germany) according to the manufacturer's instructions.

Strain UCH007 was also co-cultured with Sulfurospirillum cavolei UCH003 [13] in bicarbonate-buffered medium for 36 days. Cells were harvested from $100 \mathrm{~mL}$ of the culture by centrifugation $\left(12,000 \times g, 15 \mathrm{~min}, 4{ }^{\circ} \mathrm{C}\right)$. Total DNA was extracted using the DNeasy Blood and Tissue Kit (Qiagen) according to the manufacturer's instructions. The effects of strain UCH003 on the growth of strain UCH007, will be described in a separate report (manuscript in preparation).

\section{Genome sequencing and assembly}

It was difficult to obtain sufficient genomic DNA for direct shotgun sequencing from the pure culture of strain UCH007. It was also difficult to construct a complete genome sequence using reads generated by WGA because of the high abundance of chimeric reads. Therefore, direct shotgun sequencing was performed using the mixed genomic DNA obtained from the coculture. Then, the differential reads picking method (Fig. 3) was applied to pick up reads that originated from strain UCH007.

The DNA obtained by WGA was sequenced using a 454 GS FLX Titanium pyrosequencer (Roche, Basel, Switzerland), and generated 85,621 reads (WGA reads). 


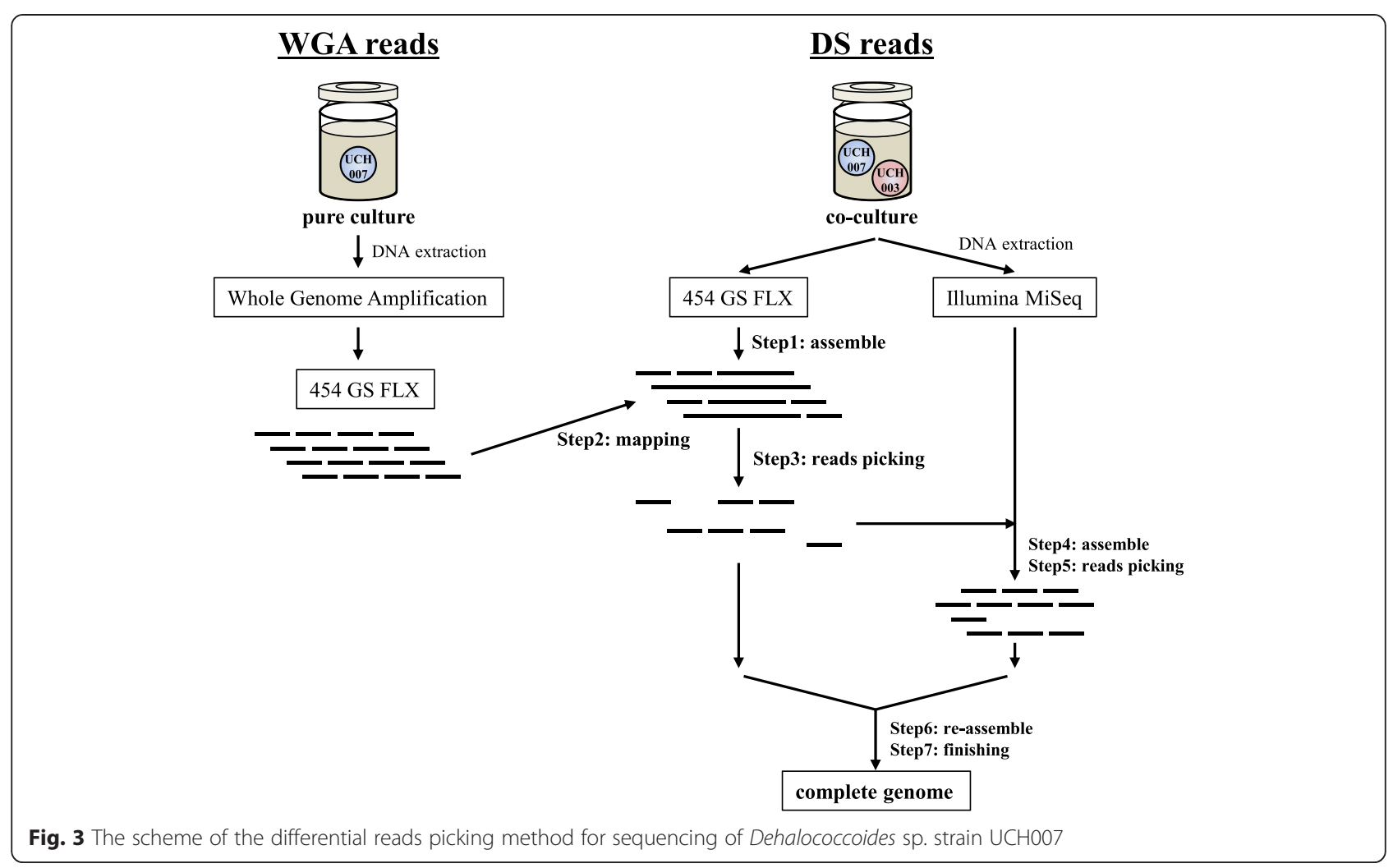

The mixed genomic DNA extracted from the co-culture was directly sequenced using 454 GS FLX and Illumina MiSeq sequencers (Illumina, San Diego, CA, USA), and generated 213,427 reads and 3,332,948 reads with $251 \mathrm{bp}$ paired-end sequencing, respectively (DS reads). The reads from the MiSeq were trimmed using sickle software with default parameters [14].

After assembling the DS reads from the 454 GS FLX using Newbler 2.6 (Roche) (Fig. 3; Step 1), the WGA reads were mapped to the resulting contigs using Newbler 2.8 (Fig. 3; Step 2). The DS reads from the 454 GS FLX that were contained in the mapped contigs were recovered, these were considered to originate from strain $\mathrm{UCH} 007$, yielding 47,262 reads $(29,841,879$ bp) (Fig. 3; Step 3). Next, these reads and 2.5 million paired-end reads and 8,414 single-end reads from the MiSeq (approximately $100 \times$ coverage against the $D$. mccartyi VS genome) were assembled using Newbler 2.6 software (Fig. 3; Step 4). Then the MiSeq reads co-assembled with the 454 GS FLX reads were picked, yielding 620,022 paired-end reads and 1,874 single-end reads $(144,540,399$ bp and $383,354 \mathrm{bp}$, respectively) (Fig. 3; Step 5). Finally, the picked DS reads both from 454 GS FLX and MiSeq were re-assembled, yielding 13 contigs (Fig. 3; Step 6). Genome closure was accomplished by manual adjustment of the assembly (Fig. 3; Step 7).

\section{Genome annotation}

The complete sequence of the chromosome was analyzed using MiGAP [15], which uses MetaGeneAnnotator [16] for predicting protein-coding genes, tRNAscan-SE [17] for tRNA genes and RNAmmer [18] for rRNA genes. The functions of the predicted protein-coding genes were assigned based on information in the Uniprot [19], Interpro [20], HAMAP [21] and KEGG [22] databases, and an in-house database composed of manually curated microbial genome sequences, as reported previously [23]. Genes in internal clusters were detected using BLASTclust with thresholds of $70 \%$ covered length and $30 \%$ sequence identity [24]. Signal peptides and transmembrane helices were predicted using SignalP [25] and TMHMM [26], respectively.

\section{Genome properties}

The genome of strain UCH007 consisted of a circular chromosome of $1,473,548$ bp with a $46.91 \% \mathrm{G}+\mathrm{C}$ content. The chromosome was predicted to contain 1,509 protein coding genes, 47 tRNA genes and 3 rRNA genes (Table 3 and Fig. 4). The distribution of protein coding genes into COG functional categories is shown in Table 4.

\section{Insights from the genome sequence}

The ANI is becoming widely accepted as a method to delineate bacterial species, with 95-96\% ANI value 
Table 3 Genome statistics

\begin{tabular}{llr}
\hline Attribute & Value & \% of total \\
\hline Genome size (bp) & $1,473,548$ & 100.00 \\
DNA coding (bp) & $1,323,945$ & 89.85 \\
DNA G+C (bp) & 691,289 & 46.91 \\
DNA scaffolds & 1 & \\
Total genes & 1,559 & 100.00 \\
Protein coding genes & 1,509 & 96.79 \\
RNA genes & 50 & 3.21 \\
Pseudo genes & 2 & 0.13 \\
Genes in internal clusters & 311 & 19.95 \\
Genes with function prediction & 1,006 & 64.53 \\
Genes assigned to COGs & 1,150 & 73.77 \\
Genes with Pfam domains & 1,224 & 78.51 \\
Genes with signal peptides & 129 & 8.27 \\
Genes with transmembrane helices & 345 & 22.13 \\
CRISPR repeats & 1 & \\
\hline
\end{tabular}

corresponding to $70 \%$ DNA relatedness [27, 28]. Löffler et al. noted that strains BAV1, CBDB1 and GT (Pinellas subgroup) showed lower ANI values, 86-87\%, to strain VS (Victoria subgroup) and strain 195 (Cornell subgroup) [1]. However, they proposed only one species, $D$. mccartyi, to accommodate all six isolates belonging to three different subgroups because of the high similarity of gene contents, and morphological and physiological characteristics. We recalculated ANI values, based on ANIb using the JSpecies program with default settings, to make full use of the accumulating genomic sequences of Dehalococcoides. The results showed that strain UCH007 was closely related to strains GY50, CG1 and VS (Victoria subgroup) with 98.52, 97.99 and $97.07 \%$ ANI values, respectively (Additional file 1: Table S1), which were above the species threshold [27]. By comparison, the strain $\mathrm{UCH} 007$ and other members of Victoria subgroup were more distantly related to strains $195^{\mathrm{T}}$ and CG4 (Cornell subgroup) with ANI values of 89.20-89.40\%, and other strains (Pinellas subgroup)

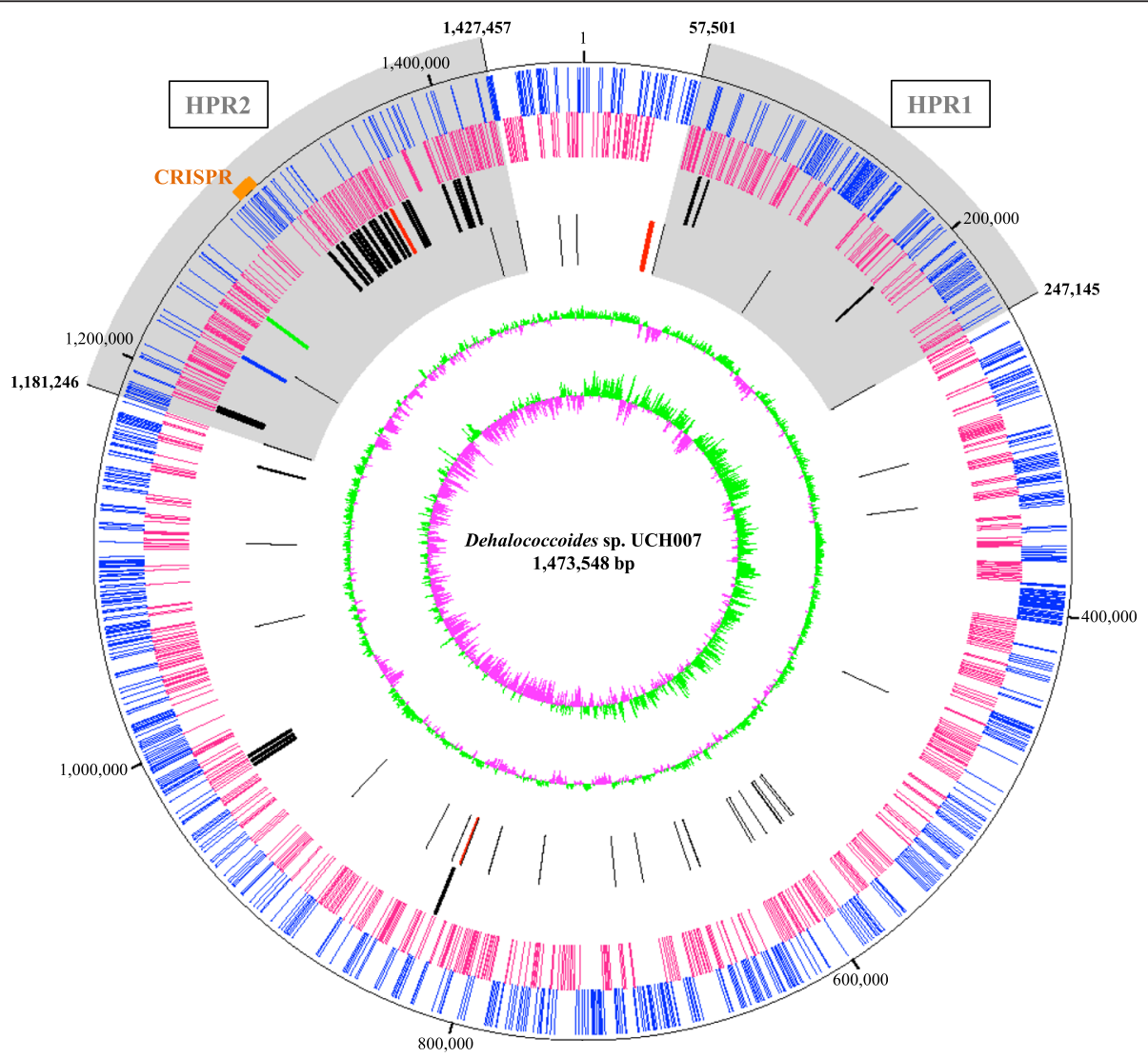

Fig. 4 Graphical circular map of the genome of Dehalococcoides sp. strain UCH007. The map was drawn using ArcWithColor [38]. From outside to the center: genes on the forward strand, genes on the reverse strand, rdhA genes (pceA gene, red; tceA gene, blue; vcrA gene, green), RNA genes (rRNAs, red; tRNAs, black), GC content, GC skew 
Table 4 Number of genes associated with general COG functional categories

\begin{tabular}{llll}
\hline Code & Value & \% of total & Description \\
\hline A & 132 & 8.75 & Translation \\
K & 0 & 0.00 & RNA processing and modification \\
L & 100 & 6.63 & Transcription \\
B & 92 & 6.10 & Replication, recombination and repair \\
D & 2 & 0.13 & Chromatin structure and dynamics \\
V & 14 & 0.93 & Cell cycle control, mitosis and meiosis \\
T & 18 & 1.19 & Defense mechanisms \\
M & 36 & 3.71 & Signal transduction mechanisms \\
N & 10 & 2.25 & Cell wall/membrane biogenesis \\
U & 28 & 0.66 & Cell motility \\
O & 54 & 3.86 & Intracellular trafficking and secretion \\
& & & Posttranslational modification, protein \\
C & 136 & 9.01 & Energy production and conversion \\
G & 41 & 2.72 & Carbohydrate transport and metabolism \\
E & 118 & 7.82 & Amino acid transport and metabolism \\
F & 49 & 3.25 & Nucleotide transport and metabolism \\
H & 70 & 4.64 & Coenzyme transport and metabolism \\
I & 28 & 1.86 & Lipid transport and metabolism \\
P & 57 & 3.78 & Inorganic ion transport and metabolism \\
Q & 10 & 0.66 & Secondary metabolites biosynthesis, \\
& & & transport and catabolism \\
R & 131 & 8.68 & General function prediction only \\
S & 98 & 6.49 & Function unknown \\
- & 359 & 23.79 & Not in CoGs \\
\hline & & &
\end{tabular}

with ANI values of $85.95-86.96 \%$. In addition, the ANI values between the strain $195^{\mathrm{T}}$ or CG4, and strains in the Pinellas subgroup were 85.22-86.02\%. Altogether, all strains in each of three subgroups, each subgroup consisting of at least two strains, showed ANI values lower than the 95-96 \% threshold to all strains in other two subgroups (Additional file 1: Table S1). These results suggest that three subgroups of Dehalococcoides are to be considered three separate species [27].

The genome of strain UCH007 harbors $29 r d h A$ and $r d h B$ gene clusters, and four of these $29 \mathrm{RdhA}$ proteins (UCH007_00760, UCH007_09900, UCH007_09930 and UCH007_13640) showed low similarities $(<55 \%)$ to those in other strains. HPRs have been designated on the genomes of strains within the genus Dehalococcoides $[9,29,30]$, and three and $22 \mathrm{rdhA}$ genes in strain UCH007 locate in HPR1 and HPR2, respectively (Fig. 4). Strain BTF08, belonging to the Pinellas subgroup, was the first strain reported to contain the $p c e A$, tce $A$ and $v c r A$ genes, encoding key enzymes in the reductive dechlorination of chloroethenes [9]. Strain UCH007 also contains orthologues of pceA (UCH007_13880), tceA (UCH007_12670) and vcrA (UCH007_12960), and is the first example of a strain containing these genes in the Victoria subgroup (Additional file 2: Table S2). The $v c r A$ gene of strain $\mathrm{UCH007}$ was detected in a genomic island located downstream of the ssrA gene as is the case with other Dehalococcoides strains $[9,31]$.

Clustered Regularly Interspaced Short Palindromic Repeat (CRISPR)-associated genes are detected on the HPR2 in the genome of strain UCH007 (UCH007_13260-13330), and 40 spacer regions (start position: 1,300,493 bp, end position: 1,302,966 bp) are predicted using the CRISPRfinder program online [32]. CRISPR-associated genes have only ever been found in the Pinellas subgroup, and strains CBDB1, DCMB5 and GT $[9,29,30]$, so this is the first report of a CRISPR region in the Victoria subgroup. A bidirectional BLASTP search of the CRISPR-associated proteins showed sequence identity of more than $73 \%$ between strain UCH007 and other strains (Additional file 3: Table S3). The direct repeat was $29 \mathrm{bp}$ in length, and the consensus sequence (5'-GTATTCCCCACGCgTGTGGG GGTGAACCG-3') was conserved among the four strains, with the exception of the base shown in lowercase [32]. Therefore, these CRISPRs seem to share a common evolutionary origin.

\section{Conclusions}

Here we reported the isolation and complete genome sequence of Dehalococcoides strain UCH007, which can dechlorinate chloroethenes to ethene. The genome sequence showed that the strain UCH007 is the first strain in the Victoria subgroup of Dehalococcoides revealed to possess $p c e A$, tce $A$ and $\nu c r A$ genes on the chromosome. As this strain is currently considered to be used in the bioaugmentation of chloroethenes-contaminated groundwater, this information will be useful for monitoring and improve the bioaugmentation process through, for example, metagenomic and metatranscriptomic analyses.

\section{Additional files}

Additional file 1: Table S1. Phylogenetic analysis using whole genome sequences and 165 rRNA genes. (XLSX $12 \mathrm{~kb}$ )

Additional file 2: Table S2. Comparison of reductive dehalogenases of strain UCH007 with those of other strains. (XLSX $12 \mathrm{~kb}$ )

Additional file 3: Table S3. Comparison of CRISPR-associated proteins of strain UCH007 with those of other strains. (XLSX $10 \mathrm{~kb}$ )

\section{Abbreviations}

ANI: average nucleotide identities; ANIb: average nucleotide identities by BLAST; cis-1,2-DCE: cis-1,2-dichloroethene; HPR: high plasticity region; MiGAP: microbial genome annotation pipeline; PCE: tetrachloroethene; RDase: reductive dehalogenase; TCE: trichloroethene; VC: vinyl chloride; WGA: whole genome amplification. 


\section{Competing interests}

The authors declare that they have no competing interests.

\section{Authors' contributions}

YU, TM and AY contributed to the conception and design of the study. YU and TM drafted the manuscript (These authors made equal contributions to this work). YU isolated Dehalococcoides sp. UCH007. TM analyzed the data. $\mathrm{AH}$ worked on genome sequencing and assembly. SO annotated the genome. MI, YT, KS and NF supervised the study. All authors read and approved the final manuscript.

\section{Acknowledgements}

This work was supported by grants from the Ministry of Economy, Trade and Industry of Japan. The authors would like to thank Dr. Moriyuki Hamada (Biological Resource Center, National Institute of Technology and Evaluation) for technical support during electron microscopy work.

\section{Author details}

${ }^{1}$ Biological Resource Center, National Institute of Technology and Evaluation, 2-10-49 Nishihara, Tokyo 151-0066, Japan. ${ }^{2}$ Taisei Corporation, 344-1 Nase, Kanagawa 245-0051, Japan.

Received: 15 May 2015 Accepted: 2 November 2015

Published online: 14 November 2015

\section{References}

1. Löffler FE, Yan J, Ritalahti KM, Adrian L, Edwards EA, Konstantinidis KT, et al. Dehalococcoides mccartyi gen. nov., sp. nov., obligately organohaliderespiring anaerobic bacteria relevant to halogen cycling and bioremediation, belong to a novel bacterial class, Dehalococcoidia classis nov., order Dehalococcoidales ord. nov. and family Dehalococcoidaceae fam. nov., within the phylum Chloroflexi. Int J Syst Evol Microbiol. 2013;63:625-35.

2. Maymó-Gatell X, Chien Y, Gossett JM, Zinder SH. Isolation of a bacterium that reductively dechlorinates tetrachloroethene to ethene. Science. 1997;276:1568-71.

3. He J, Ritalahti KM, Yang KL, Koenigsberg SS, Löffler FE. Detoxification of vinyl chloride to ethene coupled to growth of an anaerobic bacterium. Nature. 2003;424:62-5.

4. He J, Sung Y, Krajmalnik-Brown R, Ritalahti KM, Löffler FE. Isolation and characterization of Dehalococcoides sp. strain FL2, a trichloroethene (TCE)and 1,2-dichloroethene-respiring anaerobe. Environ Microbiol. 2005;7:1442-50.

5. Sung Y, Ritalahti KM, Apkarian RP, Löffler FE. Quantitative PCR confirms purity of strain GT, a novel trichloroethene-to-ethene-respiring Dehalococcoides isolate. Appl Environ Microbiol. 2006;72:1980-7.

6. Müller JA, Rosner BM, Von Abendroth G, Meshulam-Simon G, McCarty PL, Spormann AM. Molecular identification of the catabolic vinyl chloride reductase from Dehalococcoides sp. strain VS and its environmental distribution. Appl Environ Microbiol. 2004;70:4880-8.

7. Cheng $D$, He J. Isolation and characterization of "Dehalococcoides" sp. strain $\mathrm{MB}$, which dechlorinates tetrachloroethene to trans-1,2-dichloroethene. Appl Environ Microbiol. 2009;75:5910-8.

8. Lee PK, Cheng D, Hu P, West KA, Dick GJ, Brodie EL, et al. Comparative genomics of two newly isolated Dehalococcoides strains and an enrichment using a genus microarray. ISME J. 2011;5:1014-24.

9. Pöritz M, Goris T, Wubet T, Tarkka MT, Buscot F, Nijenhuis I, et al. Genome sequences of two dehalogenation specialists - Dehalococcoides mccartyi strains BTF08 and DCMB5 enriched from the highly polluted Bitterfeld region. FEMS Microbiol Lett. 2013;343:101-4.

10. Wang S, Chng KR, Wilm A, Zhao S, Yang KL, Nagarajan N, et al. Genomic characterization of three unique Dehalococcoides that respire on persistent polychlorinated biphenyls. Proc Natl Acad Sci USA. 2014;111:12103-8.

11. Miura T, Yamazoe A, Ito M, Ohji S, Hosoyama A, Takahata Y, et al. The impact of injections of different nutrients on bacterial community and its dechlorination activity in chloroethene-contaminated groundwater. Microbes Environ. 2015;30:164-71.

12. Field D, Garrity G, Gray T, Morrison N, Selengut J, Sterk P, et al. The minimum information about a genome sequence (MIGS) specification. Nat Biotechnol. 2008;26:541-7.

13. Miura T, Uchino Y, Tsuchikane K, Ohtsubo Y, Ohji S, Hosoyama A, et al. Complete genome sequence of Sulfurospirillum strain UCH0O1 and UCH003 isolated from groundwater in Japan. Genome Announc. 2015;3:e00236-15.
14. najoshi/sickle [https://github.com/najoshi/sickle]. Access date 6/11/2015.

15. Microbial Genome Annotation Pipeline [http://www.migap.org/index.php/ en]. Access date 6/11/2015.

16. Noguchi H, Taniguchi T, Itoh T. MetaGeneAnnotator: detecting speciesspecific patterns of ribosomal binding site for precise gene prediction in anonymous prokaryotic and phage genomes. DNA Res. 2008;15:387-96.

17. Lowe TM, Eddy SR. tRNAscan-SE: a program for improved detection of transfer RNA genes in genomic sequence. Nucleic Acids Res. 1997;25:955-64.

18. Lagesen $K$, Hallin P, Rødland EA, Staerfeldt HH, Rognes T, Ussery DW. RNAmmer: consistent and rapid annotation of ribosomal RNA genes. Nucleic Acids Res. 2007;35:3100-8.

19. UniProt Consortium. The universal protein resource (UniProt) in 2010. Nucleic Acids Res. 2010;38:D142-8.

20. Mulder NJ, Apweiler R, Attwood TK, Bairoch A, Bateman A, Binns D, et al. New developments in the InterPro database. Nucleic Acids Res. 2007;35:D224-8.

21. Lima T, Auchincloss AH, Coudert E, Keller G, Michoud K, Rivoire C, et al. HAMAP: a database of completely sequenced microbial proteome sets and manually curated microbial protein families in UniProtKB/Swiss-Prot. Nucleic Acids Res. 2009;37:D471-8.

22. Kanehisa M, Araki M, Goto S, Hattori M, Hirakawa M, Itoh M, et al. KEGG for linking genomes to life and the environment. Nucleic Acids Res. 2007;36:D480-4.

23. Shintani M, Hosoyama A, Ohji S, Tsuchikane K, Takarada H, Yamazoe A, et al. Complete genome sequence of the carbazole degrader Pseudomonas resinovorans strain CA10 (NBRC 106553). Genome Announc. 2013;1:e00488-13.

24. BLASTclust [http://toolkit.tuebingen.mpg.de/blastclust]. Access date 6/11/ 2015.

25. SignalP [http://www.cbs.dtu.dk/services/SignalP/]. Access date 6/11/2015.

26. TMHMM. Transmembrane domain prediction. [http://www.cbs.dtu.dk/ services/TMHMM/]. Access date 6/11/2015.

27. Richter M, Rosselló-Móra R. Shifting the genomic gold standard for the prokaryotic species definition. Proc Natl Acad Sci U S A. 2009;106:19126-31.

28. Goris J, Konstantinidis KT, Klappenbach JA, Coenye T, Vandamme P, Tiedje JM. DNA-DNA hybridization values and their relationship to whole-genome sequence similarities. Int J Syst Evol Microbiol. 2007;57:81-91.

29. McMurdie PJ, Behrens SF, Müller JA, Göke J, Ritalahti KM, Wagner R, et al. Localized plasticity in the streamlined genomes of vinyl chloride respiring Dehalococcoides. PLoS Genet. 2009;5:e1000714.

30. Kube M, Beck A, Zinder SH, Kuhl H, Reinhardt R, Adrian L. Genome sequence of the chlorinated compound-respiring bacterium Dehalococcoides species strain CBDB1. Nat Biotechnol. 2005;23:1269-73.

31. McMurdie PJ, Hug LA, Edwards EA, Holmes S, Spormann AM. Site-specific mobilization of vinyl chloride respiration islands by a mechanism common in Dehalococcoides. BMC Genomics. 2011;12:287.

32. UUU CRISPRfinder program online [http://crispr.u-psud.fr/Server/]. Access date 6/11/2015.

33. Tamura K, Peterson D, Peterson N, Stecher G, Nei M, Kumar S. MEGA5: molecular evolutionary genetics analysis using maximum likelihood, evolutionary distance, and maximum parsimony methods. Mol Biol Evol. 2011;28:2731-9.

34. Woese CR, Kandler O, Wheelis ML. Towards a natural system of organisms: proposal for the domains Archaea, Bacteria, and Eucarya. Proc Natl Acad Sci U S A. 1990;87:4576-9.

35. Castenholz RW. Class I: "Chloroflexi". In: Boone DR, Castenholz RW, Garrity GM, editors. Bergey's Manual of Systematic Bacteriology, vol. Volume 1. Secondth ed. New York: Springer; 2001. p. 427.

36. Oren A, Garrity GM. List of new names and new combinations previously effectively, but not validly, published. Int J Syst Evol Microbiol. 2013;63:3131-4.

37. Ashburner M, Ball CA, Blake JA, Botstein D, Butler H, Cherry JM, et al. Gene ontology: tool for the unification of biology. The Gene Ontology Consortium. Nat Genet. 2000;25:25-9.

38. Ohtsubo Y, Ikeda-Ohtsubo W, Nagata Y, Tsuda M. GenomeMatcher: a graphical user interface for DNA sequence comparison. BMC Bioinformatics. 2008;9:376. 